\title{
Long-term effect of sitagliptin on endothelial function in type 2 diabetes: a sub-analysis of the PROLOGUE study
}

\author{
Tatsuya Maruhashi ${ }^{1}$, Yukihito Higashi ${ }^{2,3^{*}}$, Yasuki Kihara ${ }^{1}$, Hirotsugu Yamada ${ }^{4}$, Masataka Sata ${ }^{5}$, \\ Shinichiro Ueda ${ }^{6}$, Masato Odawara ${ }^{7}, Y^{2}$ asuo Terauchi ${ }^{8}$, Kazuoki Dai ${ }^{9}$, Jun Ohno ${ }^{10}$, Masato lida ${ }^{11}$, Hiroaki Sano ${ }^{12}$, \\ Hirofumi Tomiyama ${ }^{13}$, Teruo Inoue ${ }^{14}$, Atsushi Tanaka ${ }^{15}$, Toyoaki Murohara ${ }^{16}$, Koichi Node $^{15}$ \\ and for the PROLOGUE Study Investigators
}

\begin{abstract}
Background: As a sub-analysis of the PROLOGUE study, we evaluated the long-term effect of sitagliptin, a dipeptidyl peptidase 4 inhibitor, on endothelial function in the conduit brachial artery in patients with type 2 diabetes.

Methods: In the PROLOGUE study, patients were randomly assigned to either add-on sitagliptin treatment (sitagliptin group) or continued conventional antihyperglycemic treatment (conventional group). Among the 463 participants in the PROLOGUE study, FMD was measured in 17 patients in the sitagliptin group and 18 patients in the conventional group at the beginning and after 12 and 24 months of treatment.

Results: $\mathrm{HbA} 1 \mathrm{c}$ levels were significantly decreased after 12 and 24 months of treatment compared to baseline values in both groups ( $7.0 \pm 0.4$ vs. $6.6 \pm 0.3$ and $6.6 \pm 0.4 \%$ in the sitagliptin group; $7.0 \pm 0.6$ vs. $6.6 \pm 0.7$ and $6.6 \pm 0.7 \%$ in the conventional group; $P<0.05$, respectively). There was no significant difference between FMD values at baseline and after 12 and 24 months in the sitagliptin group ( $4.3 \pm 2.6$ vs. $4.4 \pm 2.1$ and $4.4 \pm 2.3 \%, P=1.0$, respectively). Although FMD had a tendency to increase from $4.3 \pm 2.4 \%$ at baseline to $5.2 \pm 1.9 \%$ after 12 months and $5.1 \pm 2.2 \%$ after 24 months in the conventional group, there was no significant difference between FMD values at baseline and after 12 and 24 months ( $P=0.36$ and 0.33 , respectively).

Conclusions: Add-on sitagliptin to conventional antihyperglycemic drugs in patients with type 2 diabetes did not alter endothelial function in the conduit brachial artery measured by FMD during a 2-year study period. Sitagliptin may be used without concern for an adverse effect on endothelial function in patients with type 2 diabetes.

Trial registration: University hospital Medical Information Network (UMIN) Center: ID UMIN000004490
\end{abstract}

Keywords: Dipeptidyl peptidase 4 inhibitor, Flow-mediated vasodilation, Type 2 diabetes

\section{Background}

Endothelial dysfunction is the initial step in the pathogenesis of atherosclerosis and plays an important role in the development of this condition $[1,2]$. In addition, it has been shown that endothelial function is an

\footnotetext{
*Correspondence: yhigashi@hiroshima-u.ac.jp

${ }^{3}$ Department of Cardiovascular Regeneration and Medicine, Research Institute for Radiation Biology and Medicine, Hiroshima University, 1-2-3 Kasumi, Minami-ku, Hiroshima 734-8551, Japan

Full list of author information is available at the end of the article
}

independent predictor of cardiovascular events [3]. Type 2 diabetes, an important risk factor for cardiovascular disease, is associated with endothelial dysfunction $[4,5]$. Several investigators have reported that lifestyle modification and pharmacological therapy, including antihyperglycemic agents, improve endothelial function in patients with type 2 diabetes [6-9]. These findings suggest that endothelial dysfunction is reversible and can be restored through an appropriate intervention in patients with type 2 diabetes. 
An orally administered dipeptidyl peptidase 4 (DPP-4) inhibitor is now available for treatment of type 2 diabetes. The DPP-4 inhibitor prolongs the action of incretin hormones by inhibition of their breakdown and improves glycemic control through incretin hormone-induced decrease in glucagon levels and increase in endogenous insulin secretion in patients with type 2 diabetes. The relationship between treatment with a DPP-4 inhibitor and endothelial function in patients with type 2 diabetes has been evaluated [10-13]. Sitagliptin, a DPP-4 inhibitor, has been demonstrated to significantly improve microvascular endothelial function assessed by the reactive hyperemia peripheral arterial tonometry index after 6 months in uncontrolled diabetic patients with coronary heart disease [10]. As for the relationship between a DPP-4 inhibitor and endothelial function in the conduit brachial artery assessed by flow-mediated vasodilation (FMD), a previous short-term study demonstrated that 6-week treatment with DPP-4 inhibitors, including sitagliptin and alogliptin, attenuated FMD [11], whereas other previous studies demonstrated that 12-week treatment with sitagliptin improved FMD in patients with type 2 diabetes $[12,13]$. However, the long-term effect of a DPP-4 inhibitor on FMD in patients with type 2 diabetes remains unclear.

The PROLOGUE study was a prospective multicenter study conducted to evaluate the inhibitory effect of a DPP-4 inhibitor on progression of atherosclerosis based on carotid artery intima-media thickness (IMT) assessed by ultrasonography over a 2-year follow-up period [14]. In that study, FMD in the brachial artery was also measured in some of the subjects. Therefore, we carried out the present study as a sub-analysis of the PROLOGUE study to evaluate the long-term effect of a DPP-4 inhibitor on endothelial function assessed by FMD in the brachial artery in patients with type 2 diabetes.

\section{Methods}

\section{Study design and patients}

The rationale and design of the PROLOGUE study (University Hospital Medical Information Network Center: ID 000004490) have been described previously [15]. In brief, the PROLOGUE study was a multicenter, prospective, randomized, open-label trial and blinded-endpoint trial carried out with the participation of 48 Japanese institutions. Eligible patients were at least 30 years of age and who had type 2 diabetes with HbA1c level of 6.2$9.4 \%$ despite conventional treatment with diet, exercise and/or pharmacologic therapy with oral antihyperglycemic agents (except incretin-related therapy) for more than 3 months. Patients who had taken a DPP-4 inhibitor, glucagon-like peptide-1 (GLP-1) analogs, or insulin before randomization were excluded. Other exclusion criteria are described elsewhere [15].
Between June 2011 and September 2012, a total of 463 patients with type 2 diabetes were enrolled and randomly assigned in a 1:1 ratio to either add-on sitagliptin treatment (sitagliptin group: $\mathrm{n}=232$ ) or conventional antihyperglycemic treatment (conventional group: $\mathrm{n}=231$ ). The treatment randomization was conducted on basis of the age, gender, use of statins, pre-treatment diabetic type (non-pharmacological or pharmacological treatment), HbA1c ( $<7$ or $\geq 7 \%$ ), office systolic blood pressure $(<135$ or $\geq 135 \mathrm{~mm} \mathrm{Hg})$, and maximum IMT $(<1.0$ or $\geq 1.0 \mathrm{~mm}$ ) [15]. All patients were treated with the aim of achieving a targeted HbA1c level less than $6.2 \%$ or fasting plasma glucose level less than $110 \mathrm{mg} / \mathrm{dL}$ during the study period. Treatment of patients in the sitagliptin group was initially started with sitagliptin at a dose of $50 \mathrm{mg}$ daily. If further glycemic intervention was necessary, the dose of sitagliptin was increased up to $100 \mathrm{mg}$ daily within 3 months, and conventional antihyperglycemic agents other than DPP-4 inhibitors, GLP-1 analogs and/or insulin were added. If further glycemic intervention was necessary in patients in the conventional group, antihyperglycemic agents other than DPP-4 inhibitors, GLP-1 analogs and/or insulin were added. All of the patients were followed up annually for 2 years until September 2014.

In the PROLOGUE study, the primary endpoint was the change in mean common carotid artery-IMT at 24 months after treatment. Carotid ultrasound examinations were performed at the beginning of treatment and after 12 and 24 months of treatment. The secondary outcomes included changes in FMD in the brachial artery after 12 and 24 months of treatment [15]. In some of the participating institutions, FMD in the brachial artery was also measured as an optional examination. Of a total of 463 patients, serial measurement of FMD was performed in 17 patients in the sitagliptin group and 18 patients in the conventional group at the beginning and after 12 and 24 months of treatment. The data for these 35 patients from 4 institutions were analyzed in the present study. This sub-study is a pre-specified analysis. The ethical committees of the participating institutions approved the study protocol. Written informed consent for participation in the study was obtained from all subjects.

\section{Study protocol}

All studies were performed in the morning, after overnight fasting, in a quiet, dark, and air-conditioned room (constant temperature of $22-25{ }^{\circ} \mathrm{C}$ ). The subjects were kept in the supine position throughout the study. A 23-gauge polyethylene catheter was inserted into the left deep antecubital vein to obtain blood samples. The vascular response to reactive hyperemia in the brachial artery was used for the assessment of 
endothelium-dependent FMD. The observers were blind to the form of examination.

\section{Measurement of FMD}

The same protocol for measurement of FMD in the brachial artery was used in the study. FMD was measured using the same ultrasound instrument specialized for FMD measurements in all institutions. A high-resolution linear artery transducer was coupled to computerassisted analysis software (UNEXEF18G, UNEX Co, Nagoya, Japan) that used an automated edge detection system for measurement of brachial artery diameter. A blood pressure cuff was placed around the forearm. The brachial artery was scanned longitudinally $5-10 \mathrm{~cm}$ above the elbow. When the clearest B-mode image of the anterior and posterior intimal interfaces between the lumen and vessel wall was obtained, the transducer was held at the same point throughout the scan by a special probe holder (UNEX Co) to ensure consistency of the image. Depth and gain setting were set to optimize the images of the arterial lumen wall interface. When the tracking gate was placed on the intima, the artery diameter was automatically tracked, and the waveform of diameter changes over the cardiac cycle was displayed in real time using the FMD mode of the tracking system. This allowed the ultrasound images to be optimized at the start of the scan and the transducer position to be adjusted immediately for optimal tracking performance throughout the scan. Pulsed Doppler flow was assessed at baseline and during peak hyperemic flow, which was confirmed to occur within $15 \mathrm{~s}$ after cuff deflation. Blood flow velocity was calculated from the color Doppler data and was displayed as a waveform in real time. The baseline longitudinal image of the artery was acquired for $30 \mathrm{~s}$, and then the blood pressure cuff was inflated to $50 \mathrm{~mm} \mathrm{Hg}$ above systolic pressure for $5 \mathrm{~min}$. The longitudinal image of the artery was recorded continuously until 5 min after cuff deflation. Pulsed Doppler velocity signals were obtained for $20 \mathrm{~s}$ at baseline and for $10 \mathrm{~s}$ immediately after cuff deflation. Changes in brachial artery diameter were immediately expressed as percentage change relative to the vessel diameter before cuff inflation. FMD was automatically calculated as the percentage change in peak vessel diameter from the baseline value. Percentage of FMD [(Peak diameter - Baseline diameter)/Baseline diameter] was used for analysis. Blood flow volume was calculated by multiplying the Doppler flow velocity (corrected for the angle) by heart rate and vessel crosssectional area $\left(-r^{2}\right)$. Reactive hyperemia was calculated as the maximum percentage increase in flow after cuff deflation compared with baseline flow. Inter- and intracoefficients of variation for the brachial artery diameter were 1.6 and $1.4 \%$, respectively.

\section{Statistical analysis}

Results are presented as mean $\pm \mathrm{SD}$. All reported probability values were 2 -sided, and a probability value of $<0.05$ was considered statistically significant. Categorical variables were compared by means of the Chi square test. We compared mean values of continuous variables between the 2 groups by unpaired Student's $t$ test. Differences in mean values of continuous variables between baseline, 12 and 24 months were compared by paired Student's $t$ test with Bonferroni's correction. The data were processed using the software package Stata version 9 (Stata Co., College Station, Texas, USA).

\section{Results}

\section{Baseline clinical characteristics}

Table 1 shows the baseline clinical characteristics of all patients and the effects of each treatment on baseline parameters in the sitagliptin group and conventional group. Of the 35 patients, 20 (57.1\%) were men and 15 (42.9\%) were women. Twenty-six (74.3\%) had hypertension, 25 (71.4 \%) had dyslipidemia, 5 (19.2\%) were current smokers, 18 (51.4\%) had coronary heart disease, and $3(8.5 \%)$ had cerebrovascular disease. The mean fasting plasma glucose level was $7.04 \pm 1.11 \mathrm{mmol} / \mathrm{L}$ and the mean HbA1c level was $7.0 \pm 0.5 \%$. The mean value of FMD was $4.3 \pm 2.4 \%$. There was no significant difference in any of the variables except the prevalence of current smokers between the two groups. Although serum levels of creatinine and lipids did not significantly change during the treatment period, systolic blood pressure was significantly higher after 24 months in the sitagliptin group than in the conventional group.

\section{Glycemic control}

$\mathrm{HbA1c}$ and fasting plasma glucose levels were similar at baseline between the two groups. HbA1c levels were significantly decreased after 12 and 24 months of treatment compared to baseline values in both groups $(7.0 \pm 0.4$ vs. $6.6 \pm 0.3$ and $6.6 \pm 0.4 \%$ in the sitagliptin group; $7.0 \pm 0.6$ vs. $6.6 \pm 0.7$ and $6.6 \pm 0.7 \%$ in the conventional group; P $<0.05$, respectively, Fig. 1a). No significant difference in fasting plasma glucose level was observed during the study period in either group (Fig. 1b).

\section{Endothelial function}

Effects of glycemic intervention on FMD at baseline and after 12 and 24 months of treatment in the sitagliptin group and conventional group are shown in Fig. 2. FMD values were similar at baseline in the two groups. There was no significant difference between FMD values at baseline and after 12 and 24 months in the sitagliptin group $(4.3 \pm 2.6$ vs. $4.4 \pm 2.1$ and $4.4 \pm 2.3 \%, \mathrm{P}=1.0$, respectively). Although FMD rose from $4.3 \pm 2.4 \%$ at 
Table 1 Clinical characteristics of the subjects

\begin{tabular}{|c|c|c|c|c|c|c|c|}
\hline \multirow[t]{2}{*}{ Variables } & \multirow{2}{*}{$\begin{array}{l}\text { All }(n=35) \\
0 \text { month }\end{array}$} & \multicolumn{3}{|c|}{ Conventional group $(n=18)$} & \multicolumn{3}{|c|}{ Sitagliptin group $(n=17)$} \\
\hline & & 0 month & 12 months & 24 months & 0 month & 12 months & 24 months \\
\hline Age, y & $66.5 \pm 8.9$ & $64.1 \pm 10.3$ & & & $69.1 \pm 6.5$ & & \\
\hline Male, n (\%) & $20(57.1)$ & $11(61.1)$ & & & $9(52.9)$ & & \\
\hline Body mass index, $\mathrm{kg} / \mathrm{m}^{2}$ & $27.0 \pm 4.2$ & $27.2 \pm 5.0$ & $27.1 \pm 4.9$ & $27.0 \pm 4.8$ & $26.8 \pm 3.3$ & $26.9 \pm 3.3$ & $26.5 \pm 3.0$ \\
\hline $\begin{array}{l}\text { Systolic blood pressure, } \\
\mathrm{mm} \mathrm{Hg}\end{array}$ & $128.0 \pm 13.1$ & $127.2 \pm 14.0$ & $129.5 \pm 15.3$ & $123.6 \pm 12.5$ & $136.9 \pm 16.7$ & $138.6 \pm 15.6$ & $133.2 \pm 13.3^{*}$ \\
\hline $\begin{array}{l}\text { Diastolic blood pressure, } \\
\mathrm{mm} \mathrm{Hg}\end{array}$ & $72.8 \pm 10.1$ & $78.8 \pm 10.6$ & $75.4 \pm 10.9$ & $72.4 \pm 10.2$ & $79.8 \pm 8.9$ & $78.5 \pm 8.8$ & $76.5 \pm 8.8$ \\
\hline Heart rate, bpm & $67.3 \pm 9.5$ & $67.1 \pm 8.6$ & $67.7 \pm 10.3$ & $67.9 \pm 8.5$ & $67.2 \pm 9.6$ & $65.6 \pm 10.7$ & $68.0 \pm 12.9$ \\
\hline Creatinine, $\mu \mathrm{mol} / \mathrm{L}$ & $72.0 \pm 19.5$ & $71.8 \pm 19.3$ & $70.5 \pm 19.6$ & $79.5 \pm 24.6$ & $72.2 \pm 20.2$ & $72.2 \pm 22.7$ & $76.8 \pm 24.3$ \\
\hline Total cholesterol, mmol/L & $4.66 \pm 0.75$ & $4.74 \pm 0.87$ & $4.95 \pm 1.19$ & $4.72 \pm 0.98$ & $4.57 \pm 0.60$ & $4.57 \pm 0.78$ & $4.60 \pm 0.69$ \\
\hline Triglycerides, mmol/L & $1.38 \pm 0.48$ & $1.49 \pm 0.47$ & $1.55 \pm 0.53$ & $1.19 \pm 0.31$ & $1.26 \pm 0.47$ & $1.26 \pm 0.65$ & $1.43 \pm 0.97$ \\
\hline HDL cholesterol, mmol/L & $1.42 \pm 0.37$ & $1.38 \pm 0.45$ & $1.44 \pm 0.40$ & $1.45 \pm 0.37$ & $1.47 \pm 0.27$ & $1.46 \pm 0.33$ & $1.47 \pm 0.34$ \\
\hline Glucose, mmol/L & $7.04 \pm 1.11$ & $7.03 \pm 1.04$ & $7.14 \pm 1.46$ & $6.66 \pm 1.67$ & $7.05 \pm 1.21$ & $6.66 \pm 1.27$ & $6.48 \pm 0.81$ \\
\hline $\mathrm{HbA} 1 \mathrm{c}, \%$ & $7.0 \pm 0.5$ & $7.0 \pm 0.6$ & $6.6 \pm 0.7$ & $6.6 \pm 0.7$ & $7.0 \pm 0.4$ & $6.6 \pm 0.3$ & $6.6 \pm 0.4$ \\
\hline $\begin{array}{l}\text { Brachial artery diameter, } \\
\text { mm }\end{array}$ & $4.08 \pm 0.55$ & $4.05 \pm 0.60$ & $4.02 \pm 0.60$ & $3.98 \pm 0.58$ & $4.11 \pm 0.52$ & $4.14 \pm 0.59$ & $4.19 \pm 0.67$ \\
\hline Current smoker, n (\%) & $5(19.2)$ & $5(41.7)$ & & & $0(0)^{*}$ & & \\
\hline \multicolumn{8}{|l|}{ Complications } \\
\hline Hypertension, n (\%) & $26(74.3)$ & $13(72.2)$ & & & $13(76.5)$ & & \\
\hline Dyslipidemia, n (\%) & $25(71.4)$ & $11(61.1)$ & & & $14(82.4)$ & & \\
\hline $\begin{array}{l}\text { Coronary heart disease, } \\
\text { n (\%) }\end{array}$ & $18(51.4)$ & $8(44.4)$ & & & $10(58.8)$ & & \\
\hline $\begin{array}{l}\text { Cerebrovascular disease, } \\
\text { n (\%) }\end{array}$ & $3(8.5)$ & $2(11.1)$ & & & $1(5.9)$ & & \\
\hline \multicolumn{8}{|l|}{ Antidiabetic drugs } \\
\hline Sulfonylurea, n (\%) & $10(28.6)$ & $5(27.8)$ & $6(33.0)$ & $6(33.0)$ & $5(29.4)$ & $3(17.6)$ & $3(17.6)$ \\
\hline Metformin, n (\%) & $9(25.7)$ & $5(27.8)$ & $7(38.9)$ & $7(38.9)$ & $4(23.5)$ & $5(29.4)$ & $5(29.4)$ \\
\hline $\begin{array}{l}\text { a-Glucosidase inhibitor, } \\
\text { n (\%) }\end{array}$ & $13(37.1)$ & $4(22.2)$ & $8(44.4)$ & $8(44.4)$ & $9(52.9)$ & $6(35.3)$ & $6(35.3)$ \\
\hline Pioglitazone, n (\%) & $4(11.4)$ & $2(11.1)$ & $3(16.7)$ & $3(16.7)$ & $2(11.8)$ & $1(5.9)$ & $1(5.9)$ \\
\hline Glinide, n (\%) & $1(2.9)$ & $0(0)$ & $1(5.6)$ & $1(5.6)$ & $1(5.9)$ & $0(0)$ & $0(0)$ \\
\hline \multicolumn{8}{|l|}{ Antihyperlipidemic drugs } \\
\hline Statin, n (\%) & $21(60.0)$ & $8(44.4)$ & $8(44.4)$ & $9(50.0)$ & $13(76.4)$ & $12(70.1)$ & $12(70.1)$ \\
\hline Fibrate, n (\%) & $2(5.7)$ & $2(11.1)$ & $2(11.1)$ & $2(11.1)$ & $0(0)$ & $0(0)$ & $0(0)$ \\
\hline Eicosapentaenoic acid, n (\%) & $2(5.7)$ & $1(5.6)$ & $1(5.6)$ & $1(5.6)$ & $1(5.9)$ & $1(5.9)$ & $1(5.9)$ \\
\hline Ezetimibe, n (\%) & $1(2.9)$ & $0(0)$ & $0(0)$ & $0(0)$ & $1(5.9)$ & $1(5.9)$ & $1(5.9)$ \\
\hline \multicolumn{8}{|l|}{ Antihypertensive drugs } \\
\hline $\begin{array}{l}\text { Calcium channel blocker, } \\
\text { n (\%) }\end{array}$ & $21(60.0)$ & $11(61.1)$ & $11(61.1)$ & $11(61.1)$ & $10(58.8)$ & $10(58.8)$ & $10(58.8)$ \\
\hline ARB, n (\%) & $20(57.1)$ & $11(61.1)$ & $11(61.1)$ & $11(61.1)$ & $9(52.9)$ & $10(58.8)$ & $10(58.8)$ \\
\hline ACE inhibitor, n (\%) & $5(14.2)$ & $3(16.7)$ & $3(16.7)$ & $3(16.7)$ & $2(11.8)$ & $1(5.9)$ & $1(5.9)$ \\
\hline Diuretic, n (\%) & $8(22.9)$ & $3(16.7)$ & $3(16.7)$ & $4(22.2)$ & $5(29.4)$ & $5(29.4)$ & $5(29.4)$ \\
\hline Beta-blocker, n (\%) & $8(22.9)$ & $4(22.2)$ & $4(22.2)$ & $4(22.2)$ & $4(23.5)$ & $5(29.4)$ & $5(29.4)$ \\
\hline \multicolumn{8}{|l|}{ Others } \\
\hline Antiplatelet agent, n (\%) & $18(51.4)$ & $7(38.9)$ & $7(38.9)$ & $7(38.9)$ & $11(64.7)$ & $12(70.6)$ & $12(70.6)$ \\
\hline
\end{tabular}

$H D L$ high-density lipoprotein; $A R B$ angiotensin receptor blockers; $A C E$ angiotensin converting enzyme * $\mathrm{P}<0.05$ vs. control group 

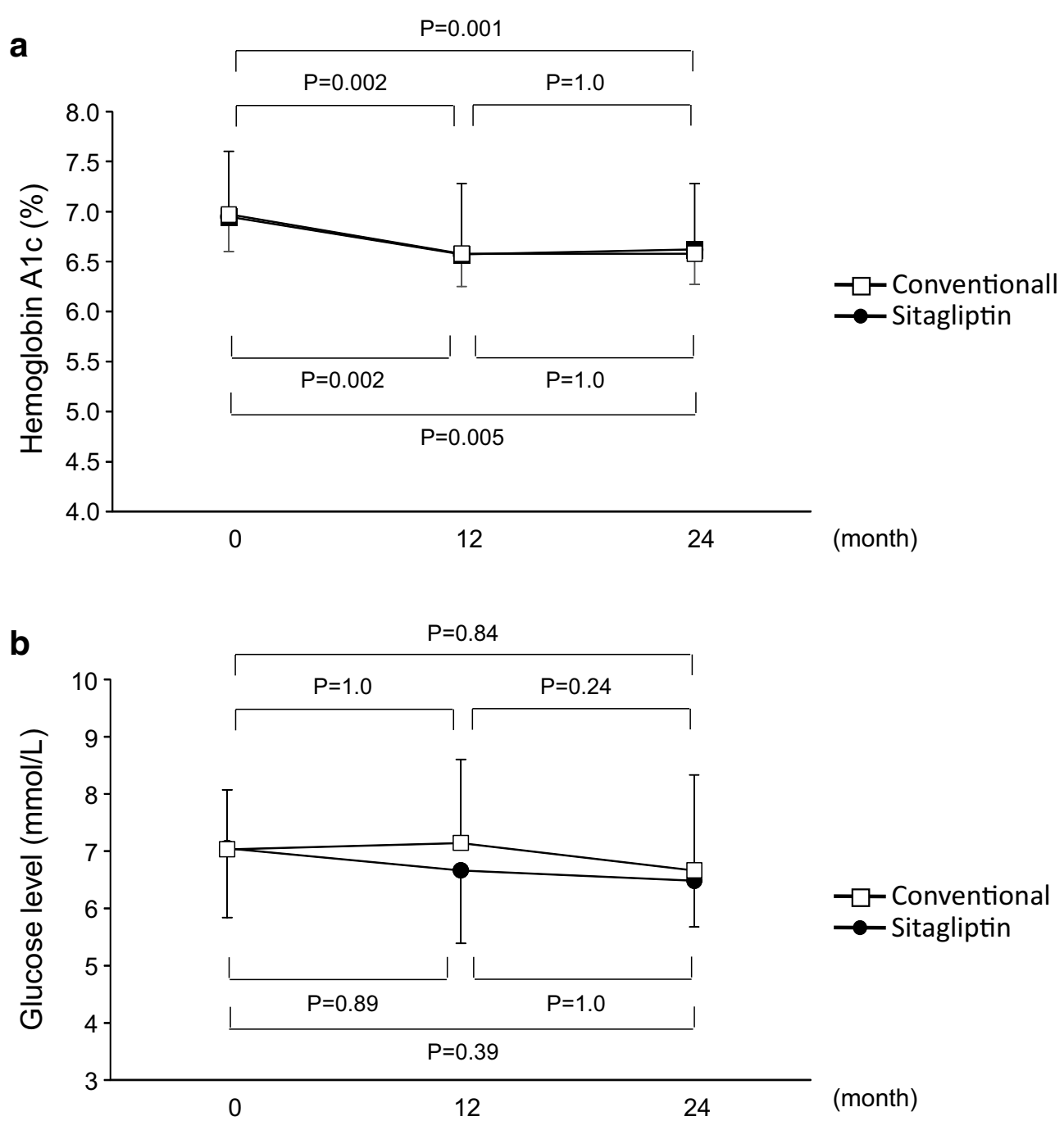

Fig. 1 Line graphs show hemoglobin A1c level (a) and fasting glucose level (b) at each study visit in the sitagliptin group and conventional group

baseline to $5.2 \pm 1.9 \%$ after 12 months and $5.1 \pm 2.2 \%$ after 24 months in the conventional group, there was no significant difference between FMD values at baseline and after 12 and 24 months $(\mathrm{P}=0.36$ and 0.33 , respectively). There was no significant difference between the two groups in FMD after 12 and 24 months $(\mathrm{P}=0.22$ and 0.31 , respectively).

\section{Discussion}

In the present study, similar degrees of improvement in glycemic control were achieved in the sitagliptin group and the conventional group. The present study demonstrated that the addition of sitagliptin to usual care in patients with type 2 diabetes did not alter endothelial function assessed by FMD in the conduit brachial artery over a 2-year study period.
In the present study, patients who had taken a DPP-4 inhibitor, GLP-1 analogs, or insulin before randomization were excluded. Moreover, additional use of incretinrelated antihyperglycemic agents and insulin for further glycemic intervention was inhibited in the conventional group during the study period according to the study protocol. Therefore, the control treatment did not mask any true effect of sitagliptin in this study.

Short-term effects of treatment with sitagliptin on FMD have been controversially reported [11-13]. Ayaori et al. [11] demonstrated that 6-week sitagliptin therapy significantly attenuated FMD despite improved diabetic status, whereas two other previous studies demonstrated that 12-week sitagliptin therapy significantly improved FMD [12, 13], suggesting that at least 12 weeks of treatment with sitagliptin is necessary for improvement of 


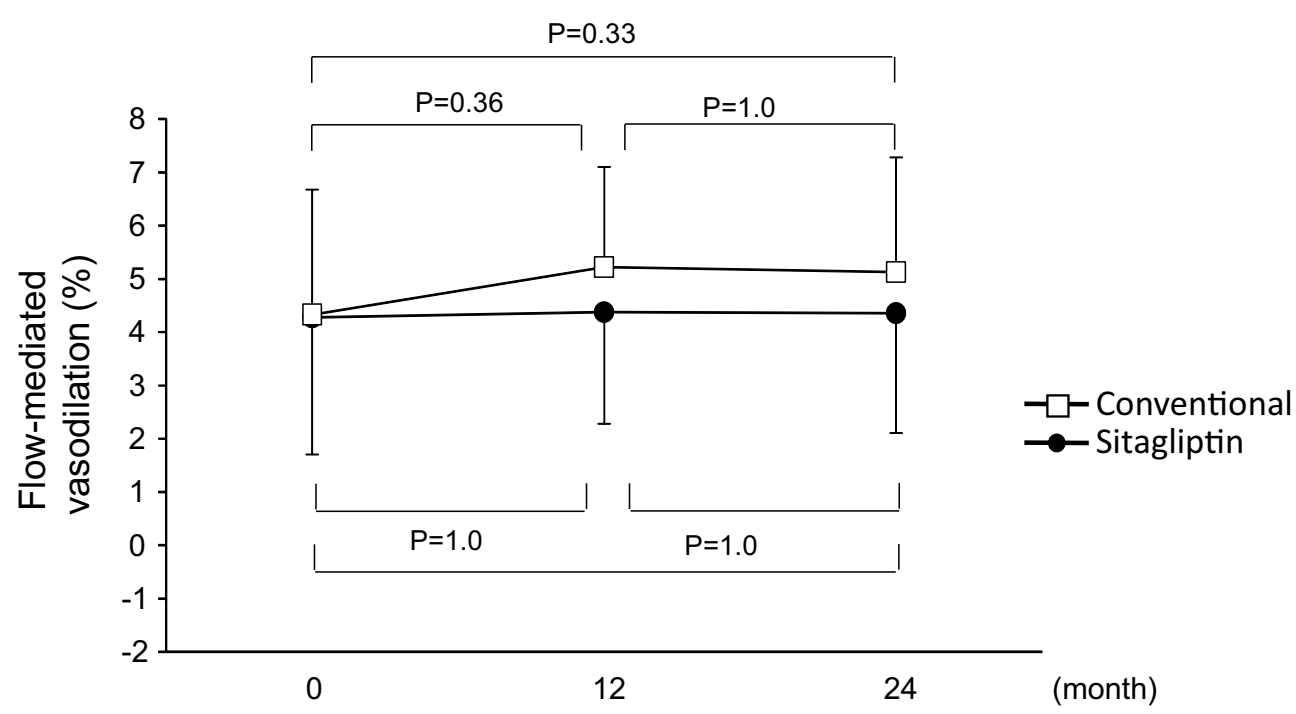

Fig. 2 Line graphs show flow-mediated vasodilation at each study visit in the sitagliptin group and conventional group

endothelial function. However, the long-term effect of sitagliptin therapy on FMD has remained unclear. In the present study, we demonstrated that FMD was not altered after 12 and 24 months. FMD was maintained at a similar level during the study period by treatment with sitagliptin in patients with type 2 diabetes. Recently, a cardiovascular safety concern regarding the long-term use of some antihyperglycemic agents has been raised $[16,17]$. Therefore, new antihyperglycemic agents are required not only to show glucose-lowering ability but also to be not associated with increases in major adverse cardiovascular events [18]. A cardiovascular effect of sitagliptin has been shown in experimental and clinical studies $[19,20]$. In an experimental model, it was demonstrated that sitagliptin can reduce the area of atherosclerotic lesions, possibly by regulating the AMPK and MAPK pathways and then reducing leukocyte-endothelial cell interaction and inflammation reactions [19]. Moreover, sitagliptin treatment has neutral effects on left ventricular diastolic function in diabetic patients [20]. A recent study demonstrated that adding sitagliptin to usual care in patients with both type 2 diabetes and established cardiovascular disease did not increase the risk of major adverse cardiovascular events or hospitalization for heart failure during a median follow-up period of 3.0 years [21]. In the secondary analysis of the study, it was demonstrated that sitagliptin does not affect the risk of hospitalization for heart failure in patients with type 2 diabetes, both overall and among high-risk patient subgroups [22]. These results are supported by our finding that 2-year add-on sitagliptin therapy was not associated with impairment of endothelial function in the conduit brachial artery assessed by FMD, an independent predictor of cardiovascular events.

In the conventional group, FMD increased, but not significantly, from $4.3 \pm 2.4 \%$ at baseline to $5.2 \pm 1.9 \%$ after 12 months and $5.1 \pm 2.2 \%$ after 24 months. In accordance with the study protocol, the use of antihyperglycemic agents other than DPP-4 inhibitors, GLP-1 analogs and/ or insulin was encouraged as required, with the aim of achieving the target $\mathrm{HbA1c}$ level in the conventional group during the study period. Several studies have shown that some antihyperglycemic agents have beneficial effects on endothelial function. Treatment with metformin, pioglitazone, or an $\alpha$-glucosidase inhibitor in patients with type 2 diabetes has been demonstrated to improve endothelial function assessed by FMD [13, 23-26]. Patients in the conventional group received additional antihyperglycemic agents, including metformin, $\alpha$-glucosidase inhibitor and pioglitazone, instead of sitagliptin added in the sitagliptin group, to achieve the target HbA1c level. The addition of these antihyperglycemic agents might have contributed to the increasing tendency in FMD in the conventional group.

\section{Limitations}

A major limitation of this study is a small sample size. The present study was a sub-analysis of the PROLOGUE study, and the number of study subjects was relatively small. Unfortunately, there is no sample size for power calculation since FMD was a voluntary measurement parameter in the PROLOGUE trial, and this may be underpowered. Further studies enrolling a large number of subjects are needed to confirm the long-term effect of a DPP-4 inhibitor on endothelial function in patients with type 2 diabetes. 


\section{Conclusions}

Adding sitagliptin to usual care in patients with type 2 diabetes did not alter endothelial function in the conduit brachial artery measured by FMD during a 2-year study period. Sitagliptin may be used in patients with type 2 diabetes without concern for an adverse effect on endothelial function (Additional file 1).

\section{Additional file}

Additional file 1. All dataset supporting the conclusions of this article.

\section{Abbreviations}

DPP-4: dipeptidyl peptidase 4; FMD: flow-mediated vasodilation; GLP-1: glicagon-like peptide-1; IMT: intima-media thickness.

\section{Authors' contributions}

$\mathrm{TMa}, \mathrm{YH}$, and $\mathrm{KN}$, drafting the article and conception of this study; TMa, HY, MS, SU, MO, YH, KD, JO, MI, HS, HT, TI, and AT, recruiting study participants; YK and $\mathrm{TMu}$, revising the article critically for important intellectual content. $\mathrm{YH}$ is the guarantor of this work and, as such, had full access to all the data in the study and takes responsibility for the integrity of the data and the accuracy of the data analysis. All authors read and approved the final manuscript.

\begin{abstract}
Author details
${ }^{1}$ Department of Cardiovascular Medicine, Graduate School of Biomedical and Health Sciences, Hiroshima University, 1-2-3 Kasumi, Minami-ku, Hiroshima 734-8551, Japan. ${ }^{2}$ Division of Regeneration and Medicine, Medical Center for Translational and Clinical Research, Hiroshima University Hospital, 1-2-3 Kasumi, Minami-ku, Hiroshima 734-8551, Japan. ${ }^{3}$ Department of Cardiovascular Regeneration and Medicine, Research Institute for Radiation Biology and Medicine, Hiroshima University, 1-2-3 Kasumi, Minami-ku, Hiroshima 734-8551, Japan. ${ }^{4}$ Department of Cardiovascular Medicine, Tokushima University Hospital, 2-50-1 Kuramoto-cho, Tokushima 770-0042 Japan. ${ }^{5}$ Department of Cardiovascular Medicine, Institute of Biomedical Sciences, Tokushima University Graduate School, 3-18-15 Kuramoto-cho, Tokushima 770-0042, Japan. ${ }^{6}$ Department of Clinical Pharmacology and Therapeutics, University of the Ryukyus, 207 Uehara, Nishihara-cho, Okinawa 903-0215, Japan. ${ }^{7}$ Department of Diabetes, Endocrinology, Metabolism and Rheumatology, Tokyo Medical University, 6-1-1 Nishishinjuku, Shinjuku-ku, Tokyo 160-0023, Japan. ${ }^{8}$ Department of Endocrinology and Metabolism, Yokohama City University, 3-9 Fukuura, Kanazawa-ku, Yokohama 236-0004, Japan. ${ }^{9}$ Department of Cardiology, Hiroshima City Hospital, 7-33 Motomachi, Naka-ku, Hiroshima 730-0011, Japan. ${ }^{10}$ Department of Cardiology, Tsushima Municipal Hospital, 3-73 Tachibana-cho, Tsushima 496-0038, Japan. ${ }^{11}$ Department of Cardiology, Mitsubishi Nagoya Hospital, 7-8 Sotodoi-cho, Atsuta-ku, Nagoya 456-0013, Japan. ${ }^{12}$ Department of Cardiology, Nagoya Ekisaikai Hospital, 4-66, Syounen-cho, Nakagawa-ku, Nagoya 454-0854, Japan. ${ }^{13}$ Department of Cardiology, Tokyo Medical University, 6-7-1, Nishishinjuku, Shinjuku-ku, Tokyo 160-0023, Japan. ${ }^{14}$ Department of Cardiovascular Medicine, Dokkyo Medical University, 880 Kitakobayashi, Mibumachi, Shimotsuga-gun, Tochigi 321-0293, Japan. ${ }^{15}$ Department of Cardiovascular Medicine, Saga University, 5-1-1, Nabeshima, Saga 849-0937, Japan. ${ }^{16}$ Department of Cardiology, Nagoya University Graduate School of Medicine, 65 Tsurumai-cho, Shouwa-ku, Nagoya 466-0065, Japan.
\end{abstract}

\section{Acknowledgements}

The authors gratefully acknowledge the technical assistance of Satoko Michiyama. The authors gratefully thank Akira Yamashina, Takanori Yasu, and Mitsuyoshi Urashima for their key roles in the independent data monitoring board.

\section{Prologue study investigators}

The PROLOGUE study is a multicenter collaboration. In addition to the listed authors, the following PROLOGUE Study Investigators were involved in this study: Masayoshi Ajioka (Department of Cardiovascular Internal Medicine, Tosei General Hospital); Toru Aoyama (Cardiology Center, Nagoya Kyoritsu
Hospital); Tetsuya Babazono (Department of Medicine, Diabetes Center, Tokyo Women's Medical University School of Medicine); Yasuko K. Bando (Department of Cardiology, Nagoya University Graduate School of Medicine and National Hospital Organization Nagoya Medical Center); Hiroyuki Daida (Department of Cardiovascular Medicine, Juntendo University Graduate School of Medicine); Jun Fukui (Division of Cardiology, Hokusho Central Hospital); Kumiko Hamano (Department of Diabetes and Endocrinology, Kanto Rosai Hospital); Shigemasa Hashimoto (Department of Cardiology, Karatsu Red Cross Hospital); Kazunori Hayashi (Department of Cardiology, Nakatsugawa Municipal Hospital); Tsutomu Hirano (Department of Diabetes, Metabolism, and Endocrinology, Showa University School of Medicine); Hideki Horibe (Department of Cardiovascular Medicine, Gifu Prefectural Tajimi Hospital); Kazuo Ibaraki (Department of Internal Medicine, Karatsu Red Cross Hospital); Takako lino (Department of Cardiovascular and Respiratory Medicine, Akita University Graduate School of Medicine); Kenji lino (Department of Cardiovascular and Respiratory Medicine, Akita University Graduate School of Medicine); Yutaka Ishibashi (Department of General Medicine, Shimane University Faculty of Medicine); Yuko S. Ishiguro (Department of Cardiology, Mitsubishi Nagoya Hospital); Masaharu Ishihara (Division of Cardiovascular Medicine and Coronary Heart Disease, Hyogo College of Medicine); Ryoji Ishiki (Division of Internal Medicine, Toyota Memorial Hospital); Tomoko Ishizu (Department of Clinical Laboratory Medicine, Faculty of Medicine, University of Tsukuba); Hiroshi Ito (Department of Cardiovascular and Respiratory Medicine, Akita University Graduate School of Medicine); Masaaki Ito (Department of Cardiology and Nephrology, Mie University Graduate School of Medicine); Yoshito Iwama (Department of Cardiology, Meijo Hospital); Hideo Izawa (Department of Cardiology, Fujita Health University Banbuntane Hotokukai Hospital); Kohei Kaku (Department of Internal Medicine, Kawasaki Medical School); Haruo Kamiya (Division of Cardiology, Japanese Red Cross Nagoya Daiichi Hospital); Kenshi Kan (Division of Diabetes, Metabolism and Endocrinology, Tokyo Medical University Hospital); Naoki Kashihara (Department of Nephrology and Hypertension, Kawasaki Medical School); Akira Kimura (Department of Cardiology, Meijo Hospital, Federation of National Public Service Personnel Mutual Aid Association); Ichiro Kishimoto (Department of Atherosclerosis and Diabetes, National Cerebral and Cardiovascular Center); Kazuo Kitagawa (Department of Neurology, Tokyo Women's Medical University); Masafumi Kitakaze (Department of Clinical Medicine and Development, National Cerebral and Cardiovascular Center); Tomoki Kitano (Department of Cardiology, National Hospital Organization Nagoya Medical Center); Yoshihisa Kizaki (Department of Cardiology, Sasebo Chuo Hospital); Kenji Kohara (Division of Diabetes, Endocrinology and Metabolism, Kawasaki Medical School); Hiroshi Koiwaya (Department of Cardiology, Miyazaki Medical Association Hospital); Taizo Kondo (Department of Cardiology, Gifu Prefectural Tajimi Hospital); Toshimitsu Kosaka (Department of Cardiovascular and Respiratory Medicine, Akita University Graduate School of Medicine); Nehiro Kuriyama (Department of Cardiology, Miyazaki Medical Association Hospital); Shigetaka Kuroki (Eguchi Hospital); Koji Maemura (Department of Cardiovascular Medicine, Graduate School of Biomedical Sciences, Nagasaki University); Hiroaki Masuzaki (Second Department of Medicine, Division of Endocrinology, Diabetes and Metabolism, Hematology, Rheumatology, Graduate School of Medicine, University of the Ryukyus); Munehide Matsuhisa (Diabetes Therapeutics and Research Center, Tokushima University); Kaori Miwa (Department of Neurology and Stroke Center, Osaka University Graduate School of Medicine); Takashi Miwa (Department of Diabetes, Endocrinology, Metabolism and Rheumatology, Tokyo Medical University); Tetsuro Miyazaki (Department of Cardiovascular Medicine, Juntendo University School of Medicine); Kazutaka Mori (Department of Cardiology, Nagoya Medical Center); Tomoatsu Mune (Division of Diabetes, Endocrinology and Metabolism, Kawasaki Medical School); Ikue Nakadaira (Diabetes and Endocrinology, Kanto Rosai Hospital); Mashio Nakamura (Department of Cardiology and Nephrology, Mie University Graduate School of Medicine); Yoshihito Nakashima (Department of Cardiovascular Disease, Tosei General Hospital); Masayuki Nakayama (JCHO Saga Central Hospital); Mamoru Nanasato (Cardiovascular Center, Japanese Red Cross Nagoya Daini Hospital); Kosaku Nitta (Department of Medicine, Kidney Center, Tokyo Women's Medical University); Yasunori Oguma (Department of Cardiovascular and Respiratory Medicine, Akita University Graduate School of Medicine); Hirotoshi Ohmura (Department of Cardiovascular Medicine, Juntendo University Graduate School of Medicine); Shinji Okubo (Japan Labour Health and Welfare Organization, Kashima Hospital, Special Department and Cardiology, Tokyo Medical University); Jun-ichi Oyama (Department of Cardiovascular Medicine, Saga University); Sosho Ri (Division of Diabetes, Metabolism and 
Endocrinology, Internal Medicine Center, Showa University Koto Toyosu Hospital); Kenji Sadamatsu (Department of Cardiology, Saga Medical Center Koseikan); Makoto Saitoh (Department of Internal Medicine, Nishio Municipal Hospital); Masaki Sakakibara (Department of Cardiology, Handa City Hospital); Yasunori Sato (Department of Global Clinical Research, Graduate School of Medicine, Chiba University); Yoshisato Shibata (Department of Cardiology, Miyazaki Medical Association Hospital); Toshimasa Shigeta (Department of Cardiology, Gifu Prefectural Tajimi Hospital); Kenei Shimada (Department of Internal Medicine and Cardiology, Osaka City University Graduate School of Medicine); Fuji Somura (Department of Cardiology, Nagoya Central Hospital); Takashi Takei (Department of Medicine, Kidney Center, Tokyo Women's Medical University); Toshiro Tanaka (Department of Internal Medicine, Nishio Municipal Hospital); Yoshito Tanioka (Division of Cardiology, Omura Municipal Hospital); Akihiro Terasawa (Department of Cardiology, Kasugai Municipal Hospital); Masahiko Tsujii (Department of Gastroenterology, Osaka Rosai Hospital); Shuichi Tsuruoka (Department of Nephrology, Nippon Medical School); Hiroyuki Tsutsui (Department of Cardiovascular Medicine, Hokkaido University Graduate School of Medicine); Hisashi Umeda (Division of Cardiology, Toyota Memorial Hospital); Mitsuyoshi Urashima (Division of Molecular Epidemiology, Jikei University School of Medicine); Hiroki Watanabe (Department of Internal Medicine, Nishio Municipal Hospital); Masato Watarai (Cardiovascular Center, Anjo Kosei Hospital); Takaaki Yamada (Department of Cardiology, Nagoya Medical Center); Hiroshi Yamamoto (Cardiovascular Surgery, Yamamoto Memorial Hospital); Akira Yamashina (Department of Cardiology, Tokyo Medical University); Kentaro Yamashita (Department of Cardiology, Nagoya University Graduate School of Medicine and National Hospital Organization Nagoya Medical Center); Takanori Yasu (Department of Cardiovascular Medicine, Dokkyo Medical University Nikko Medical Center); Chie Yasuoka (Department of Cardiovascular Medicine, Omura Municipal Hospital); Kiyoshi Yokoi (Department of Cardiovascular Medicine, Gifu Prefectural Tajimi Hospital).

\section{Competing interests}

$\mathrm{YH}$ received honoraria and research grant from MSD and received honoraria from ONO PHARMACERTICAL CO., LTD. HY and MS received honoraria and research grant from MSD and received research grant from ONO PHARMACERTICAL CO., LTD. SU and TMu received honoraria and research grant from MSD. MO received honoraria from ONO PHARMACERTICAL CO., LTD. YT received honoraria and research grant from MSD and ONO PHARMACERTICAL CO., LTD.

\section{Availability of data and materials}

The dataset supporting the conclusions of this article is included within the article (and its Additional file 1).

\section{Ethics approval and consent to participate}

The ethical committees of the participating institutions approved the study protocol. All participants provide written informed consent before data collection.

\section{Sources of funding}

This study is supported by a research grant from the Clinical Research Promotion Foundation (No.1026). The funding body had no role in study design, data collection and analysis, decision to publish or preparation of the manuscript.

Received: 1 July 2016 Accepted: 12 August 2016 Published online: 13 September 2016

\section{References}

1. Ross R. Atherosclerosis-an inflammatory disease. N Engl J Med. 1999;340(2):115-26

2. Higashi Y, Noma K, Yoshizumi M, Kihara Y. Endothelial function and oxidative stress in cardiovascular diseases. Circ J. 2009;73(3):411-8.

3. Lerman A, Zeiher AM. Endothelial function: cardiac events. Circulation. 2005;111(3):363-8.

4. Williams SB, Cusco JA, Roddy MA, Johnstone MT, Creager MA. Impaired nitric oxide-mediated vasodilation in patients with non-insulin-dependent diabetes mellitus. J Am Coll Cardiol. 1996;27(3):567-74.
5. Henry RM, Ferreira I, Kostense PJ, Dekker JM, Nijpels G, Heine RJ, Kamp O, Bouter LM, Stehouwer CD. Type 2 diabetes is associated with impaired endothelium-dependent, flow-mediated dilation, but impaired glucose metabolism is not; the Hoorn study. Atherosclerosis. 2004;174(1):49-56.

6. Maiorana A, O'Driscoll G, Cheetham C, Dembo L, Stanton K, Goodman C, Taylor R, Green D. The effect of combined aerobic and resistance exercise training on vascular function in type 2 diabetes. J Am Coll Cardiol. 2001;38(3):860-6.

7. Hamdy O, Ledbury S, Mullooly C, Jarema C, Porter S, Ovalle K, Moussa A, Caselli A, Caballero AE, Economides PA, et al. Lifestyle modification improves endothelial function in obese subjects with the insulin resistance syndrome. Diabetes Care. 2003;26(7):2119-25.

8. Tan KC, Chow WS, Tam SC, Ai VH, Lam CH, Lam KS. Atorvastatin lowers C-reactive protein and improves endothelium-dependent vasodilation in type 2 diabetes mellitus. J Clin Endocrinol Metab. 2002;87(2):563-8.

9. Ceriello A, Taboga C, Tonutti L, Quagliaro L, Piconi L, Bais B, Da Ros R, Motz E. Evidence for an independent and cumulative effect of postprandial hypertriglyceridemia and hyperglycemia on endothelial dysfunction and oxidative stress generation: effects of short- and long-term simvastatin treatment. Circulation. 2002;106(10):1211-8.

10. Matsubara J, Sugiyama S, Akiyama E, Iwashita S, Kurokawa H, Ohba K, Maeda H, Fujisue K, Yamamoto E, Kaikita K, et al. Dipeptidyl peptidase-4 inhibitor, sitagliptin, improves endothelial dysfunction in association with its anti-inflammatory effects in patients with coronary artery disease and uncontrolled diabetes. Circ J. 2013;77(5):1337-44.

11. Ayaori M, Iwakami N, Uto-Kondo H, Sato H, Sasaki M, Komatsu T, lizuka M, Takiguchi S, Yakushiji E, Nakaya K, et al. Dipeptidyl peptidase-4 inhibitors attenuate endothelial function as evaluated by flowmediated vasodilatation in type 2 diabetic patients. J Am Heart Assoc. 2013:2(1):e003277.

12. Kubota Y, Miyamoto M, Takagi G, Ikeda T, Kirinoki-Ichikawa S, Tanaka K, Mizuno K. The dipeptidyl peptidase-4 inhibitor sitagliptin improves vascular endothelial function in type 2 diabetes. J Korean Med Sci. 2012;27(11):1364-70.

13. Nakamura K, Oe H, Kihara H, Shimada K, Fukuda S, Watanabe K, Takagi T, Yunoki K, Miyoshi T, Hirata K, et al. DPP-4 inhibitor and alpha-glucosidase inhibitor equally improve endothelial function in patients with type 2 diabetes: EDGE study. Cardiovasc Diabetol. 2014;13:110.

14. Oyama JI, Murohara T, Kitakaze M, Ishizu T, Sato Y, Kitagawa K, Kamiya H, Ajioka M, Ishihara M, Dai K, et al. The effect of sitagliptin on carotid artery atherosclerosis in type 2 diabetes: the PROLOGUE randomized controlled trial. PLoS Med. 2016;13(6):e1002051.

15. Oyama J, Ishizu T, Sato Y, Kodama K, Bando YK, Murohara T, Node K. Rationale and design of a study to evaluate the effects of sitagliptin on atherosclerosis in patients with diabetes mellitus: PROLOGUE study. Int J Cardiol. 2014;174(2):383-4.

16. Nissen SE, Wolski K. Effect of rosiglitazone on the risk of myocardial infarction and death from cardiovascular causes. N Engl J Med. 2007;356(24):2457-71.

17. Holman RR, Sourij H, Califf RM. Cardiovascular outcome trials of glucose-lowering drugs or strategies in type 2 diabetes. Lancet. 2014;383(9933):2008-17.

18. Hennekens $C H$, Hebert PR, Schneider WR, O'Brien P, Demets D, Borer JS. Academic perspectives on the United States Food and Drug Administration's guidance for industry on diabetes mellitus. Contemp Clin Trials. 2010;31(5):411-3.

19. Zeng Y, Li C, Guan M, Zheng Z, Li J, Xu W, Wang L, He F, Xue Y. The DPP-4 inhibitor sitagliptin attenuates the progress of atherosclerosis in apolipoprotein-E-knockout mice via AMPK- and MAPK-dependent mechanisms. Cardiovasc Diabetol. 2014;13:32.

20. Oe H, Nakamura K, Kihara H, Shimada K, Fukuda S, Takagi T, Miyoshi T, Hirata K, Yoshikawa J, Ito H, et al. Comparison of effects of sitagliptin and voglibose on left ventricular diastolic dysfunction in patients with type 2 diabetes: results of the 3D trial. Cardiovasc Diabetol. 2015:14:83.

21. Green JB, Bethel MA, Armstrong PW, Buse JB, Engel SS, Garg J, Josse R, Kaufman KD, Koglin J, Korn S, et al. Effect of sitagliptin on cardiovascular outcomes in type 2 diabetes. N Engl J Med. 2015;373(3):232-42. 
22. McGuire DK, Van de Werf F, Armstrong PW, Standl E, Koglin J, Green JB, Bethel MA, Cornel JH, Lopes RD, Halvorsen S, et al. Association between sitagliptin use and heart failure hospitalization and related outcomes in type 2 diabetes mellitus: secondary analysis of a randomized clinical trial. JAMA Cardiol. 2016;1(2):126-35.

23. Wu S, Li X, Zhang H. Effects of metformin on endothelial function in type 2 diabetes. Exp Ther Med. 2014;7(5):1349-53.

24. Suzuki M, Takamisawa I, Yoshimasa Y, Harano Y. Association between insulin resistance and endothelial dysfunction in type 2 diabetes and the effects of pioglitazone. Diabetes Res Clin Pract. 2007;76(1):12-7.
25. Hidaka T, Nakagawa K, Goto C, Soga J, Fujii Y, Hata T, Idei N, Fujimura N, Chayama K, Kihara Y, et al. Pioglitazone improves endotheliumdependent vasodilation in hypertensive patients with impaired glucose tolerance in part through a decrease in oxidative stress. Atherosclerosis. 2010;210(2):521-4

26. Kato T, Inoue T, Node K. Postprandial endothelial dysfunction in subjects with new-onset type 2 diabetes: an acarbose and nateglinide comparative study. Cardiovasc Diabetol. 2010;9:12.

\section{Submit your next manuscript to BioMed Central and we will help you at every step:}

- We accept pre-submission inquiries

- Our selector tool helps you to find the most relevant journal

- We provide round the clock customer support

- Convenient online submission

- Thorough peer review

- Inclusion in PubMed and all major indexing services

- Maximum visibility for your research

Submit your manuscript at www.biomedcentral com/submit 